PROCEEDINGS OF THE

AMERICAN MATHEMATICAL SOCIETY

Volume 138, Number 10, October 2010, Pages 3753-3758

S 0002-9939(10)10438-9

Article electronically published on April 22, 2010

\title{
A REMARK ON NONLINEAR DIRAC EQUATIONS
}

\author{
CHANGYOU WANG
}

(Communicated by Matthew J. Gursky)

\begin{abstract}
For an $n$-dimensional spin manifold $M$ with a fixed spin structure and a spinor bundle $\Sigma M$, we prove an $\epsilon$-regularity theorem for weak solutions to the nonlinear Dirac equation

$$
\not \partial \psi=H_{j k l}\left\langle\psi^{j}, \psi^{k}\right\rangle \psi^{l},
$$

of cubic nonlinearity. In particular, it implies that any weak solution is smooth when $n=2$, which answers a question raised by Chen, Jost, and Wang.
\end{abstract}

\section{INTRODUCTION}

Linear Dirac type equations, including the Cauchy-Riemann equation in dimension two, are the most fundamental first order system of elliptic equations. During the course of studying Dirac-harmonic maps with curvature term from a Riemann surface into a Riemannian manifold, Chen-Jost-Wang [4, 5] introduced the nonlinear Dirac equation with cubic nonlinearity:

$$
\not \partial \psi^{i}=\sum_{j, k, l=1}^{N} H_{j k l}^{i}\left\langle\psi^{j}, \psi^{k}\right\rangle \psi^{l}, 1 \leq i \leq N .
$$

In dimension two, an interesting feature of this nonlinear Dirac equation is that it is conformally invariant and has critical nonlinearity, where the classical methods fail to apply. Thus it is an interesting question to study the regularity of weak solutions of (2). The aim of this short paper is to provide a general regularity criterion for (11), that, in particular, implies the smoothness of any weak solutions when $n=2$.

In order to describe the results, we briefly review some background materials on spin manifolds. The interested readers can consult with Lawson-Michelsohn 6], Chen-Jost-Li-Wang [2, 3] for more details. For $n \geq 2$, let $(M, g)$ be a spin manifold with a given spin structure and an associated spinor bundle $\Sigma$. Let $\langle\cdot, \cdot\rangle$ be a Hermitian metric on $\Sigma$ and $\nabla$ be the Levi-Civita connection on $\Sigma$ compatible with both $\langle\cdot, \cdot\rangle$ and $g$. The Dirac operator on $M$ is defined by $\not \partial=e_{\alpha} \circ \nabla_{e_{\alpha}}$, where $\left\{e_{\alpha}\right\}_{\alpha=1}^{n}$ is a local orthonormal frame on $M$, and $\circ: T M \otimes_{\mathbb{C}} \Sigma \rightarrow \Sigma$ is the Clifford multiplication.

Received by the editors October 13, 2008 and, in revised form, January 20, 2009.

2010 Mathematics Subject Classification. Primary 58J05.

The author was partially supported by NSF grant 0601162 .

(C)2010 American Mathematical Society 
Now let's write (11) in the form

$$
\not \partial \psi=H_{j k l}\left\langle\psi^{j}, \psi^{k}\right\rangle \psi^{l},
$$

where $\psi=\left(\psi^{1}, \cdots, \psi^{N}\right) \in(\Gamma \Sigma)^{N}, N \geq 1, H_{j k l}=\left(H_{j k l}^{1}, \cdots, H_{j k l}^{N}\right) \in C^{\infty}\left(M, \mathbb{R}^{N}\right)$.

We refer the reader to [5], §1, where the authors discussed two interesting examples in which (2) arises naturally. The first example is the Dirac-harmonic map $(\phi, \psi)$ associated with the Dirac-harmonic energy functional with curvature term, a nonlinear $\sigma$-model in superstring theory, in which the nonlinear Dirac equation for $\psi$ reduces to (2) when $\phi$ is a constant map. The second example is the Weierstrass representation formula for minimal surfaces $X$ immersed in $\mathbb{R}^{3}$ by holomorphic 1forms and meromorphic functions, in which an equation of the form (2) appears naturally.

It turns out that the underlying function space for the equation (2) is $L^{4}(M)$. As pointed out by [5] that any weak solution $\psi$ of (2) is smooth provided $\psi \in L^{p}(M)$ for some $p>4$. In [5], the authors proved three interesting analytic properties of (2) for $n=2$ : (i) the gradient estimate for smooth solutions $\psi$ of (2) under the smallness condition of the $L^{4}$-norm of $\psi$, (ii) the isolated singularity removable theorem, and (iii) the energy identity theorem for sequentially weak convergent smooth solutions of (2). At the end of $\S 1$ in [5], the authors asked

Conjecture 1.1. For $n=2$, any weak solution $\psi \in L^{4}(M)$ of (2) is smooth.

In this short note, we answer Conjecture 1.1 affirmatively. In fact, we prove a general regularity theorem for weak solutions of (2) in any dimensions. The idea is based on an application of the estimate of Reisz potentials between Morrey spaces, due to Adams [1. Similar techniques have been employed in the proof of higher order regularity of Dirac-harmonic maps by Wang and $\mathrm{Xu}$ [7]. The proof turns out to be very elementary and shall be applicable to other similar problems.

Before stating our results, let's first recall the definition of weak solutions of (2).

Definition 1.2. A section $\psi \in L^{4}\left((\Gamma \Sigma)^{N}\right)$ is a weak solution of (2) if

$$
\int_{M}\langle\psi, \not \partial \eta\rangle=\int_{M} H_{j k l}\left\langle\psi^{j}, \psi^{k}\right\rangle\left\langle\psi^{l}, \eta\right\rangle
$$

holds for any smooth section $\eta \in C^{\infty}\left((\Gamma \Sigma)^{N}\right)$.

Denote by $i_{M}>0$ the injectivity radius of $M$. For $0<r<i_{M}$ and $x \in M$, denote by $B_{r}(x)$ the geodesic ball in $M$ with center $x$ and radius $r$. Now we state our theorems.

Theorem 1.3. For any $n \geq 2$, there exists $\epsilon_{0}>0$ depending on $n$ such that if $\psi \in L^{4}\left((\Gamma \Sigma)^{N}\right)$ is a weak solution of the Dirac equation (2) and satisfies, for some $x_{0} \in M$ and $0<r_{0} \leq \frac{1}{2} i_{M}$,

$$
\sup _{x \in B_{r_{0}}\left(x_{0}\right), 0<r \leq r_{0}}\left\{\frac{1}{r^{n-2}} \int_{B_{r}(x)}|\psi|^{4}\right\} \leq \epsilon_{0}^{4},
$$

then $\psi \in C^{\infty}\left(B_{\frac{r_{0}}{2}}\left(x_{0}\right)\right)$.

Note that by the Hölder inequality, we have for $n \geq 2$,

$$
\frac{1}{r^{n-2}} \int_{B_{r}(x)}|\psi|^{4} \leq\left(\int_{B_{r}(x)}|\psi|^{2 n}\right)^{\frac{2}{n}} .
$$


Thus, as an immediate consequence of Theorem 1.3. we obtain

Corollary 1.4. For $n \geq 2$, if $\psi \in L^{2 n}\left((\Gamma \Sigma)^{N}\right)$ is a weak solution of the Dirac equation (2), then $\psi \in C^{\infty}\left((\Gamma \Sigma)^{N}\right)$.

It is clear that when $n=2$, Corollary 1.4 implies Conjecture 1.1.

\section{Proof of Theorem 1.3}

This section is devoted to the proof of Theorem 1.3 Since the regularity is a local property, we assume, for simplicity of presentation, that for $x_{0} \in M$, the geodesic ball $B_{i_{M}}\left(x_{0}\right) \subset M$ with the metric $g$ is identified by $\left(B_{2}, g_{0}\right)$. Here $B_{2} \subset \mathbb{R}^{n}$ is the ball with center 0 and radius 2 , and $g_{0}$ is the Euclidean metric on $\mathbb{R}^{n}$. We also assume that $\left.\Sigma\right|_{B_{2}}=B_{2} \times \mathbb{C}^{L}$, with $L=\operatorname{rank}_{\mathbb{C}} \Sigma$.

Let's also recall the definition of Morrey spaces.

Definition 2.1. For $1 \leq p \leq n, 0<\lambda \leq n$, and a domain $U \subseteq \mathbb{R}^{n}$, the Morrey space $M^{p, \lambda}(U)$ is defined by

$$
M^{p, \lambda}(U):=\left\{f \in L_{\mathrm{loc}}^{p}(U):\|f\|_{M^{p, \lambda}(U)}<+\infty\right\},
$$

where

$$
\|f\|_{M^{p, \lambda}(U)}^{p}=\sup \left\{r^{\lambda-n} \int_{B_{r}}|f|^{p}: B_{r} \subseteq U\right\} .
$$

It is easy to see that for $1 \leq p \leq n, M^{p, \lambda}(U) \subset L^{p}(U), M^{p, n}(U)=L^{p}(U)$ and $M^{p, p}(U)$ behaves like $L^{n}(U)$ from the viewpoint of scalings.

It is clear that the condition (44) in Theorem 1.3 is equivalent to

$$
\|\psi\|_{M^{4,2}\left(B_{r_{0}}\left(x_{0}\right)\right)} \leq \epsilon_{0}
$$

Thus Theorem 1.3 follows from the following lemma.

Lemma 2.2. For any $4<p<+\infty$ and $n \geq 2$, there exists $\epsilon_{0}>0$ depending only on $p$ and $n$ such that if $\psi \in M^{4,2}\left(B_{1}\right)$ is a weak solution of (2) and

$$
\|\psi\|_{M^{4,2}\left(B_{1}\right)} \leq \epsilon_{0}
$$

then $\psi \in L^{p}\left(B_{\frac{1}{16}}, \mathbb{C}^{N L}\right)$. Furthermore, $\psi \in C^{\infty}\left(B_{\frac{1}{16}}, \mathbb{C}^{N L}\right)$ and the estimate

$$
\left\|\nabla^{l} \psi\right\|_{C^{0}\left(B_{\frac{1}{16}}\right)} \leq C\left(\epsilon_{0}, l\right), \forall l \geq 1
$$

holds.

Proof. Applying $\not \partial$ to (2), we have, for $1 \leq i \leq N$,

$$
\not \partial^{2} \psi^{i}=\not \partial\left(H_{j k l}^{i}\left\langle\psi^{j}, \psi^{k}\right\rangle \psi^{l}\right)
$$

in the sense of distributions. By Lichnerowitz's formula (cf. [6]), we have

$$
-\Delta \psi^{i}=\not \not^{2} \psi^{i}
$$

Hence we have

$$
-\Delta \psi^{i}=\not \partial\left(H_{j k l}\left\langle\psi^{j}, \psi^{k}\right\rangle \psi^{l}\right)
$$

in the sense of distributions. 
For $m=1,2$, let $\eta_{m} \in C_{0}^{\infty}\left(B_{1}\right)$ be such that $0 \leq \eta_{m} \leq 1, \eta_{m} \equiv 1$ on $B_{2^{1-2 m}}$. For $1 \leq i \leq N$, define $f_{m}^{i}: \mathbb{R}^{n} \rightarrow \mathbb{C}^{L}$ by letting

$$
f_{m}^{i}(x)=\int_{\mathbb{R}^{n}} \frac{\partial G(x, y)}{\partial y_{\alpha}} \frac{\partial}{\partial y_{\alpha}} \circ\left(\eta_{m}^{3} H_{j k l}\left\langle\psi^{j}, \psi^{k}\right\rangle \psi^{l}\right)(y) d y,
$$

where $G(x, y)$ is the fundamental solution of $\Delta$ on $\mathbb{R}^{n}$. For $1 \leq i \leq N$, define $g_{m}^{i}: B_{1} \rightarrow \mathbb{C}^{L}$ by letting

$$
\psi^{i}=f_{m}^{i}+g_{m}^{i} .
$$

Direct calculations imply that for $m=1,2$ and $1 \leq i \leq N$,

$$
\begin{aligned}
-\Delta f_{m}^{i} & =\not \partial\left(\eta_{m}^{3} H_{j k l}\left\langle\psi^{j}, \psi^{k}\right\rangle \psi^{l}\right) \\
& =\not \partial\left(H_{j k l}\left\langle\psi^{j}, \psi^{k}\right\rangle \psi^{l}\right) \text { in } B_{2^{1-2 m}} .
\end{aligned}
$$

This and (7) imply that

$$
\Delta g_{m}^{i}=0 \text { in } B_{2^{1-2 m}} .
$$

It follows from (8) that for $m=1,2$ and $1 \leq i \leq N$,

$$
\left|f_{m}^{i}\right|(x) \leq C \int_{\mathbb{R}^{n}}|x-y|^{1-n}\left(\eta_{m}(y)|\psi(y)|\right)^{3} d y=C I_{1}\left(\eta_{m}^{3}|\psi|^{3}\right)(x),
$$

where

$$
I_{1}(f)(x)=\int_{\mathbb{R}^{n}}|x-y|^{1-n}|f(y)| d y, \quad f: \mathbb{R}^{n} \rightarrow \mathbb{R},
$$

is the Riesz potential of order one. Let's recall Adams' inequality on Morrey spaces (cf. [1]):

$$
\left\|I_{1}(f)\right\|_{M^{\frac{\lambda q}{\lambda-q}, \lambda}\left(\mathbb{R}^{n}\right)} \leq C\|f\|_{M^{q, \lambda}\left(\mathbb{R}^{n}\right)}, \forall 1 \leq q<\lambda \leq n .
$$

Step $1(m=1)$. Since $\left(\eta_{1}|\psi|\right)^{3} \in M^{\frac{4}{3}, 2}\left(\mathbb{R}^{n}\right)$, (13) implies that for $1 \leq i \leq N$,

$$
\begin{aligned}
\left\|f_{1}^{i}\right\|_{M^{4,2}\left(\mathbb{R}^{n}\right)} & \leq C\left\|\eta_{1}^{3}|\psi|^{3}\right\|_{M^{\frac{4}{3}, 2}\left(\mathbb{R}^{n}\right)}=C\left\|\eta_{1} \mid \psi\right\|_{M^{4,2}\left(\mathbb{R}^{n}\right)}^{3} \\
& \leq C\|\psi\|_{M^{4,2}\left(B_{1}\right)}^{3} \leq C \epsilon_{0}^{2}\|\psi\|_{M^{4,2}\left(B_{1}\right)} .
\end{aligned}
$$

On the other hand, by the standard estimate for harmonic functions, we have that for any $\theta \in\left(0, \frac{1}{4}\right)$ and $x_{0} \in B_{\frac{1}{4}}$,

$$
\left\|g_{1}^{i}\right\|_{M^{4,2}\left(B_{\theta}\left(x_{0}\right)\right)} \leq C \theta^{\frac{1}{2}}\left\|g_{1}^{i}\right\|_{M^{4,2}\left(B_{\frac{1}{2}}\right)}, \forall 1 \leq i \leq N .
$$

Putting (14) and (15) into (9) yields that for $1 \leq i \leq N$,

$$
\begin{aligned}
\left\|\psi^{i}\right\|_{M^{4,2}\left(B_{\theta}\left(x_{0}\right)\right)} & \leq C \theta^{\frac{1}{2}}\left\|g_{1}^{i}\right\|_{M^{4,2}\left(B_{\frac{1}{2}}\right)}+C \epsilon_{0}^{2}\|\psi\|_{M^{4,2}\left(B_{1}\right)} \\
& \leq C \theta^{\frac{1}{2}}\left[\left\|\psi^{i}\right\|_{M^{4,2}\left(B_{\frac{1}{2}}\right)}+\left\|f_{1}^{i}\right\|_{M^{4,2}\left(B_{\frac{1}{2}}\right)}\right]+C \epsilon_{0}^{2}\|\psi\|_{M^{4,2}\left(B_{1}\right)} \\
& \leq C\left(\epsilon_{0}^{2}+\theta^{\frac{1}{2}}\right)\|\psi\|_{M^{4,2}\left(B_{1}\right)} .
\end{aligned}
$$

This clearly implies that for any $\theta \in\left(0, \frac{1}{4}\right)$ and $x_{0} \in B_{\frac{1}{4}}$,

$$
\|\psi\|_{M^{4,2}\left(B_{\theta}\left(x_{0}\right)\right)} \leq C\left(\epsilon_{0}^{2}+\theta^{\frac{1}{2}}\right)\|\psi\|_{M^{4,2}\left(B_{1}\right)} .
$$

For any $\alpha \in\left(0, \frac{1}{3}\right)$, first choose $\theta \in\left(0, \frac{1}{4}\right)$ such that $2 C \theta^{\frac{1}{2}} \leq \theta^{\frac{\alpha}{2}}$ and then choose $\epsilon_{0}>0$ such that $2 C \epsilon_{0}^{2} \leq \theta^{\frac{\alpha}{2}}$. Then we have

$$
\|\psi\|_{M^{4,2}\left(B_{\theta}\left(x_{0}\right)\right)} \leq \theta^{\frac{\alpha}{2}}\|\psi\|_{M^{4,2}\left(B_{1}\right)}, \forall x_{0} \in B_{\frac{1}{4}} .
$$


Iteration of (18) yields

$$
\|\psi\|_{M^{4,2}\left(B_{r}\left(x_{0}\right)\right)} \leq C r^{\frac{\alpha}{2}}\|\psi\|_{M^{4,2}\left(B_{1}\right)}, \forall x_{0} \in B_{\frac{1}{4}} \text { and } 0 \leq r<\frac{1}{4} .
$$

In particular, we have for any $0<\alpha<\frac{1}{3}$,

$$
r^{2(1-\alpha)-n} \int_{B_{r}\left(x_{0}\right)}|\psi|^{4} \leq C \int_{B_{1}}|\psi|^{4}, \forall x_{0} \in B_{\frac{1}{4}} \text { and } 0<r<\frac{1}{4} .
$$

Thus $\psi \in M^{4,2(1-\alpha)}\left(B_{\frac{1}{4}}\right)$ for any $\alpha \in(0,1)$.

Step $2(m=2)$. We want to repeat the above argument to show that $\psi \in$ $M^{\frac{4-4 \alpha}{1-3 \alpha}, 2(1-\alpha)}\left(B_{\frac{1}{16}}\right)$. In fact, since $\left(\eta_{2}|\psi|\right)^{3} \in M^{\frac{4}{3}, 2(1-\alpha)}\left(\mathbb{R}^{n}\right)$, (13) implies that $f_{2}^{i} \in M^{\frac{4(1-\alpha)}{1-3 \alpha}, 2(1-\alpha)}\left(\mathbb{R}^{n}\right)$, and

$$
\begin{aligned}
\left\|f_{2}^{i}\right\|_{M^{\frac{4(1-\alpha)}{1-3 \alpha}, 2(1-\alpha)}\left(B_{\left.\frac{1}{8}\right)}\right.} & \leq\left\|f_{2}^{i}\right\|_{M^{\frac{4(1-\alpha)}{1-3 \alpha}, 2(1-\alpha)}\left(\mathbb{R}^{n}\right)} \\
& \leq C\left\|\eta_{2}^{3}|\psi|^{3}\right\|_{M^{\frac{4}{3}, 2(1-\alpha)}\left(\mathbb{R}^{n}\right)} \\
& \leq C\|\psi\|_{M^{4,2(1-\alpha)\left(B_{\frac{1}{4}}\right)} .}
\end{aligned}
$$

On the other hand, since $g_{2}^{i}$ is a harmonic function on $B_{\frac{1}{8}}$, we have, by (21),

$$
\begin{aligned}
& \left\|g_{2}^{i}\right\|_{M^{\frac{4(1-\alpha)}{1-3 \alpha}, 2(1-\alpha)}\left(B_{\frac{1}{16}}\right)} \\
\leq & C\left\|g_{2}^{i}\right\|_{M^{\frac{4(1-\alpha)}{1-3 \alpha}, 2(1-\alpha)}\left(B_{\frac{1}{8}}\right)} \\
\leq & C\left[\left\|f_{2}^{i}\right\|_{M^{\frac{4(1-\alpha)}{1-3 \alpha}, 2(1-\alpha)}\left(B_{\left.\frac{1}{8}\right)}\right.}+\left\|\psi^{i}\right\|_{M^{\frac{4(1-\alpha)}{1-3 \alpha}, 2(1-\alpha)}\left(B_{\frac{1}{8}}\right)}\right] \\
\leq & C\|\psi\|_{M^{\frac{4(1-\alpha)}{1-3 \alpha}, 2(1-\alpha)}\left(B_{\frac{1}{8}}\right)} \cdot
\end{aligned}
$$

Putting (21) and (22) into (91) yields that $\psi \in M^{\frac{4(1-\alpha)}{1-3 \alpha}, 2(1-\alpha)}\left(B_{\frac{1}{16}}\right)$ and

$$
\|\psi\|_{M^{\frac{4(1-\alpha)}{1-3 \alpha}, 2(1-\alpha)\left(B_{\left.\frac{1}{16}\right)}\right.}} \leq C\|\psi\|_{M^{4,2(1-\alpha)\left(B_{\frac{1}{4}}\right)}} \leq C\|\psi\|_{M^{4,2}\left(B_{1}\right)} .
$$

Since

$$
\lim _{\alpha \uparrow \frac{1}{3}} \frac{4(1-\alpha)}{1-3 \alpha}=+\infty \text { and } M^{\frac{4(1-\alpha)}{1-3 \alpha}, 2(1-\alpha)}\left(B_{\frac{1}{16}}\right) \subseteq L^{\frac{4(1-\alpha)}{1-3 \alpha}}\left(B_{\frac{1}{16}}\right)
$$

it follows that $\psi \in L^{p}\left(B_{\frac{1}{16}}\right)$ for any $p>4$, and

$$
\|\psi\|_{L^{p}\left(B_{\frac{1}{16}}\right)} \leq C(n, p)\|\psi\|_{M^{4,2}\left(B_{1}\right)} .
$$

Since $|\not \partial \psi| \leq C|\psi|^{3}$, the $W^{1, p}$-estimate implies that $\psi \in W_{\text {loc }}^{1, p}\left(B_{\frac{1}{16}}, \mathbb{C}^{N L}\right)$ for any $p>4$. Hence, by the Sobolev embedding theorem, $\psi \in C^{\mu}\left(B_{\frac{1}{16}}, \mathbb{C}^{N L}\right)$ for any $\mu \in(0,1)$. By the Schauder estimate, this yields $\psi \in C^{1, \mu}\left(B_{\frac{1}{16}}, \mathbb{C}^{N L}\right)$. Hence, by the bootstrap argument, we conclude that $\psi \in C^{\infty}\left(B_{\frac{1}{16}}, \mathbb{C}^{N L}\right)$ and the estimate (5) holds. 


\section{REFERENCES}

[1] D. Adams, A note on Riesz potentials. Duke Math. J. 42 (1975), no. 4., 765-778. MR0458158 $(56: 16361)$

[2] Q. Chen, J. Jost, J. Y. Li, G. F. Wang, Dirac-harmonic maps. Math. Z. 254: 409-432 (2006). MR2262709 (2007k:58021)

[3] Q. Chen, J. Jost; J. Y. Li, G. F. Wang, Regularity theorems and energy identities for Diracharmonic maps. Math. Z. 251: 61-84 (2005). MR2176464 (2007a:58013)

[4] Q. Chen, J. Jost, G. Wang, Liouville theorems for Dirac-harmonic maps. J. Math. Phys. 48 (2007), no. 11, 113517, 13 pp. MR2370260 (2009e:58020)

[5] Q. Chen, J. Jost, G. F. Wang, Nonlinear Dirac equations on Riemann surfaces. Ann. Global Anal. Geom. 33 (2008), no. 3, 253-270. MR2390834 (2009g:58026)

[6] H. Lawson, M. L. Michelsohn, Spin geometry. Princeton University Press, 1989. MR.1031992 (91g:53001)

[7] C. Y. Wang, D. L. Xu, Regularity of Dirac-harmonic maps. Internat. Math. Res. Notices, 2009, no. 20, 3759-3792.

Department of Mathematics, University of Kentucky, Lexington, Kentucky 40506

E-mail address: cywang@ms.uky.edu 